\title{
O impacto do advento de uma Unidade Básica de Saúde Fluvial na assistência aos povos ribeirinhos do Amazonas
}

\author{
The impact of the advent of a Basic Fluvial Health Unit in assisting the riverside peoples of \\ Amazonas
}
El impacto de la llegada de una Unidad Básica de Salud Fluvial para ayudar a los pueblos ribereños de Amazonas

Marcelo Henrique da Silva Reis ${ }^{1 *}$, Jéssica Karoline Alves Portugall, Josiane Montanho Mariño ${ }^{1}$, Wendel da Silva Barros ${ }^{1}$, Jaynne de Souza Dantas ${ }^{1}$, Tanny Thaylle Gomes de Souza ${ }^{1}$, Yara da Silva dos Reis ${ }^{1}$, Sibele Naiara Ferreira Germano ${ }^{1}$, Évelyn Janaína da Silva Barão ${ }^{1}$, Duã Louise Aires de Freitas ${ }^{1}$.

\section{RESUMO}

Objetivo: Relatar o impacto que a assistência em uma Unidade Básica de Saúde Fluvial trouxe para a melhoria da assistência às populações ribeirinhas em um município do Amazonas. Relato de experiência: Trata-se de um relato de experiência do tipo descritivo, obtido durante o acompanhamento de toda logística e apresentação de relatórios de uma Unidade Básica de Saúde Fluvial do Amazonas, entre dezembro de 2017 a fevereiro de 2020. Os resultados que o advento da unidade trouxe para a população contemplam: maior cobertura dos exames citopatológicos das mulheres na faixa etária preconizada entre 25 a 64 anos, maior cobertura vacinal da população que vive mais isolada, garantia de atendimentos odontológicos para quem nunca teve acesso a esse serviço, realização de exames laboratoriais para diagnóstico imediato, além da intensificação dos serviços de prevenção, através de palestras educativas. Considerações finais: Os comunitários que vivem na área ribeirinha do município possuem carências no acesso à saúde, educação, informação, dentre outras necessidades. A estratégia de operacionalização da UBS Fluvial trouxe grandes avanços através da implementação dos programas do Ministério da Saúde, neste sentido, fortalecer e avançar nas ações da zona rural é fundamental para a cobertura destes povos.

Palavras chave: População rural, Atenção primária à saúde, Saúde pública.

\begin{abstract}
Objective: To report the impact that assistance in a Basic Fluvial Health Unit brought to the improvement of assistance to riverside populations in a municipality in Amazonas. Experience report: This is a descriptive experience report, obtained during the follow-up of all logistics and reporting of a Basic Fluvial Health Unit in Amazonas, between December 2017 and February 2020. The results that advent of the unit brought to the population include: greater coverage of cytopathological examinations of women in the recommended age range between 25 to 64 years, greater vaccination coverage of the population that lives more isolated, guarantee of dental care for those who never had access to this service, laboratory tests for immediate diagnosis, in addition to the intensification of prevention services, through educational lectures. Final considerations: The community members who live in the riverside area of the municipality lack access to health, education, information, among other needs. The operational strategy of the UBS Fluvial brought great advances through the implementation of the Ministry of Health's programs, in this sense, strengthening and advancing in the actions of the rural area is fundamental for the coverage of these peoples.
\end{abstract}

Key words: Rural population, Primary health care, Public health.

1Universidade Federal do Amazonas (UFAM), Coari - AM. *E-mail: reis.henrique.marcelo@gmail.com 


\section{RESUMEN}

Objetivo: Informar el impacto que la asistencia en una Unidad Básica de Salud Fluvial trajo a la mejora de la asistencia a las poblaciones ribereñas en un municipio de Amazonas. Informe de experiencia: Este es un informe de experiencia descriptivo, obtenido durante el seguimiento de toda la logística e informes de una Unidad Básica de Salud Fluvial en Amazonas, entre diciembre de 2017 y febrero de 2020. Los resultados que La llegada de la unidad a la población incluye: mayor cobertura de exámenes citopatológicos de mujeres en el rango de edad recomendado entre 25 y 64 años, mayor cobertura de vacunación de la población que vive más aislada, garantía de atención dental para quienes nunca tuvieron acceso a este servicio, pruebas de laboratorio para diagnóstico inmediato, además de la intensificación de los servicios de prevención, a través de conferencias educativas. Consideraciones finales: Los miembros de la comunidad que viven en la zona ribereña del municipio carecen de acceso a la salud, la educación, la información, entre otras necesidades. La estrategia operativa de UBS Fluvial trajo grandes avances a través de la implementación de los programas del Ministerio de Salud, en este sentido, fortalecer y avanzar en las acciones del área rural es fundamental para la cobertura de estos pueblos.

Palabras clave: Población rural, Atención primaria a la salud, Salud pública.

\section{INTRODUÇÃO}

O isolamento geográfico em algumas áreas da Amazônia Brasileira pode levar a exclusão social e consideráveis limitações de acesso aos serviços de saúde, significando um grande desafio para ações de saúde pública, especialmente nas áreas ribeirinhas dos municípios no interior do Amazonas (REIS MHS, et al., 2019).

A população ribeirinha presente no estado do Amazonas representa uma mistura de diferentes grupos sociais: indígenas, nordestinos, migrantes de outras regiões (FRAXE TJP, et al., 2007). Em sua grande maioria, os ribeirinhos vivem com recursos advindos da pesca e agricultura de subsistência, residindo em comunidades que geralmente estão distantes das sedes dos municípios, sendo compostas por núcleos familiares, distribuídas ao longo das margens de rios, igarapés e lagos (GAMA ASM, et al., 2018).

As comunidades ribeirinhas geralmente não possuem saneamento básico, na maioria das residências a água para consumo não é tratada e o esgoto é despejado nos quintais através de fossas rudimentares. $O$ fornecimento de energia elétrica é insatisfatório, o uso de geradores movidos a gasolina ou diesel é comum. O principal meio de transporte é a canoa movida a motor "rabeta", também são utilizadas embarcações maiores popularmente conhecidas na região como "recreios", que fazem o transporte de mercadorias e passageiros. Nas comunidades não há assistência à saúde contínua, feita apenas na sede dos municípios (SOUSA IS, 2009; GAMA ASM, et al., 2018).

A proximidade do homem com a floresta amazônica associada ao clima tropical, constituem-se como fator preponderante para o surgimento de agravos típicos da região. É comum nestas localidades a ocorrência de malária, acidentes por animais peçonhentos, doença de chagas, ferradas por arraias e outros tipos de morbidades típicas da região. O acesso aos serviços de saúde, as morbidades, dentre outras características do modo de vida dos ribeirinhos estão intimamente ligadas a variação das águas nos rios e lagos (cheias e secas). O período principal das cheias ocorre geralmente no mês de julho, com recuo das águas a partir de agosto (iniciando a estiagem), chegando aos níveis mais baixos em outubro. A partir deste período, inicia-se as chuvas e o rio avança lentamente até o mês de março, nos meses subsequentes o rio se eleva até o nível máximo (REIS MHS, et al., 2019).

Por estarem dispersos da sede dos municípios, os ribeirinhos tendem a buscar alternativas para o tratamento de eventuais problemas de saúde, seja pelo auxílio do conhecimento de pessoas mais experientes na comunidade, como curandeiros, rezadores e idosos, que utilizam plantas medicinais no tratamento de enfermidades ou pela utilização da medicina moderna, através do consumo de medicamentos industrializados, que são facilmente adquiridos em farmácias e drogarias quando deslocam-se até o município para receber benefícios ou comercializar seus produtos (GAMA ASM, 2016). 
O único profissional de saúde presente diretamente nas comunidades é o Agente Comunitário de Saúde (ACS), que precisa residir na própria comunidade e pode realizar a cobertura de até 750 pessoas conforme a necessidade (BRASIL, 2001), e em áreas endêmicas, o microscopista presta assistência na coleta, análise e diagnóstico de lâminas da malária.

Diante deste panorama, o objetivo do estudo é relatar o impacto que a assistência da Unidade Básica de Saúde Fluvial trouxe para a melhoria da assistência às populações ribeirinhas do Amazonas.

\section{RELATO DE EXPERIÊNCIA}

Trata-se de um relato de experiência do tipo descritivo, obtido durante o acompanhamento de toda logística e apresentação de relatórios em uma Unidade Básica de Saúde Fluvial em um município do estado do Amazonas, entre dezembro de 2017 a fevereiro de 2020.

Nas comunidades ribeirinhas, não há unidades de saúde e a atuação de profissionais de saúde (médicos, enfermeiros, dentistas, etc.) é esporádica, principalmente nas regiões mais longínquas. Para entender o contexto da cobertura de saúde nas populações ribeirinhas, é preciso pontuar alguns aspectos importantes ocorridos com a implementação da Política Nacional de Atenção Básica (PNAB).

A PNAB possibilitou avanços na atenção à saúde das populações ribeirinhas. A política foi implementada pelas portarias MS/GM oㅡ 2.488 e 2.490, ambas de 2011. De acordo com PNAB, os municípios da Amazônia Legal e Mato Grosso do Sul podem optar entre dois arranjos organizacionais para equipes Saúde da Família, além dos existentes para o restante do país (BRASIL, 2012):

Equipes de Saúde da Família Ribeirinhas (eSFR): desempenham a maior parte de suas funções em Unidades Básicas de Saúde construídas/localizadas nas comunidades pertencentes à área adscrita e cujo acesso se dá por meio fluvial; Equipe de Saúde da Família Fluviais (eSFF): desempenham suas funções em Unidades Básicas de Saúde Fluviais (UBSF). Em dezembro de 2017, o município foi contemplado com a moderna e confortável Unidade Básica de Saúde Fluvial, para atender os mais de 18 mil ribeirinhos que habitam as oito calhas de rio e lagos da zona rural.

Anterior a aquisição da UBS Fluvial, a Secretaria Municipal de Saúde contava com o apoio de uma grande embarcação alugada, para ser utilizada pela equipe de saúde durante as viagens de atendimento na zona rural. Além disto, pequenos botes também eram utilizados na prestação de atendimentos, principalmente quando envolviam campanhas de vacinação. Durante o período entre fevereiro a dezembro de 2017, foram realizados 11.082 atendimentos de saúde pela embarcação alugada, em 23 viagens para as diferentes calhas de rios e lagos. O número de comunidades atendidas foi de 89 , com duração máxima de dois dias para cada ação, limitando as atividades desenvolvidas. Os atendimentos realizados pela equipe de saúde estão bem descritos na Tabela 1.

Tabela 1 - Atendimentos realizados pelo Barco da Saúde de fevereiro a novembro de 2017.

\begin{tabular}{c|c|c|c|c|c|c}
\hline $\begin{array}{c}\text { Consulta } \\
\text { Médica }\end{array}$ & $\begin{array}{c}\text { Consulta de } \\
\text { Enfermagem }\end{array}$ & $\begin{array}{c}\text { Consulta } \\
\text { Odontológica }\end{array}$ & $\begin{array}{c}\text { Procedimentos } \\
\text { Técnicos }\end{array}$ & $\begin{array}{c}\text { Vacinação } \\
\text { Humana }\end{array}$ & $\begin{array}{c}\text { Vacinação } \\
\text { Antirrábica }\end{array}$ & Total \\
\hline 2.686 & 1.150 & 2.045 & 2.828 & 1.478 & 895 & 11.082 \\
$24,2 \%$ & $10,4 \%$ & $18,5 \%$ & $25,5 \%$ & $13,3 \%$ & $8,1 \%$ & $100 \%$ \\
\hline
\end{tabular}

Fonte: Reis MHS, et al., 2020.

No final de 2017, o Departamento de Atenção Básica passou a operacionalizar todos os atendimentos da população rural com suporte exclusivo da UBS Fluvial, que com sua equipe multiprofissional trabalha oferecendo os serviços de consultas médicas, atendimentos odontológicos, consultas de enfermagem, borrifação intradomiciliar, serviços de vigilância, ações de cidadania com parcerias intersetoriais, vacinação humana e antirrábica, além de realizar serviços de prevenção, através de palestras educativas na unidade e também diretamente nos centros comunitários das comunidades. Essas atividades de educação em saúde aconteciam em parceria com a Universidade Federal do Amazonas e demais profissionais que fazem 
pesquisa na região, recebendo o apoio da Secretaria de Saúde para desenvolver tais atividades com o objetivo de trazer benefícios às populações ribeirinhas.

Diferente do Barco da Saúde, a UBS Fluvial conta com uma grande e adequada estrutura física: consultório odontológico, médico, enfermagem, sala de procedimentos, sala de vacina, farmácia, laboratório para realização de exames, confortáveis dormitórios, sala de espera e triagem, para melhor acomodação da equipe e usuários durante as consultas.

Ao longo dos últimos 27 meses, entre dezembro de 2017 a fevereiro de 2020, foram realizadas 29 viagens pela UBS Fluvial, destas, 23 de grande porte, ou seja, com duração entre 12 a 15 dias ininterruptos e com a equipe diretamente embarcada em área rural. Somente neste período, foram alcançadas todas as 206 comunidades cadastradas na Secretaria Municipal de Agroeconomia, além disto, foram contempladas todas as regiões, com um total de 44.071 atendimentos de diversas especialidades Tabela 2. É importante ressaltar que os resultados que o advento da unidade trouxe para a população contemplam: maior cobertura dos exames citopatológicos das mulheres na faixa etária preconizada entre 25 a 64 anos, maior cobertura vacinal da população que vive mais isolada, garantia de atendimentos odontológicos para quem nunca teve acesso a esse serviço, realização de exames laboratoriais para diagnóstico imediato, dentre outras melhorias.

Tabela 2 - Atendimentos realizados pela UBS Fluvial de dezembro de 2017 a fevereiro de 2020.

\begin{tabular}{c|c|c|c|c|c|c|c}
\hline $\begin{array}{c}\text { Consulta } \\
\text { Médica }\end{array}$ & $\begin{array}{c}\text { Consulta de } \\
\text { Enfermagem }\end{array}$ & $\begin{array}{c}\text { Consulta } \\
\text { Odontológica }\end{array}$ & $\begin{array}{c}\text { Procedimentos } \\
\text { Técnicos }\end{array}$ & $\begin{array}{c}\text { Vacinação } \\
\text { Humana }\end{array}$ & $\begin{array}{c}\text { Vacinação } \\
\text { Antirrábica }\end{array}$ & $\begin{array}{c}\text { Exames } \\
\text { Laboratoriais }\end{array}$ & Total \\
\hline 14.553 & 3.428 & 5.210 & 5.851 & 8.598 & 1.698 & 4.733 & 44.071 \\
$33,0 \%$ & $7,8 \%$ & $11,8 \%$ & $13,3 \%$ & $19,5 \%$ & $3,9 \%$ & $10,7 \%$ & $100 \%$ \\
\hline
\end{tabular}

Fonte: Reis MHS, et al., 2020.

\section{DISCUSSÃO}

Através de suas políticas de saúde, o Sistema Único de Saúde (SUS) é o único capaz de chegar até as populações que estão mais isoladas geograficamente, garantindo a elas um de seus preceitos fundamentais que é a equidade e acesso universal às políticas de saúde. A PNAB preconiza que as equipes que fazem parte da Estratégia Saúde da Família atuem para atender os residentes na Amazônia Legal e pantanal sulmatogrossense, porém, estas equipes precisam fazer parte de arranjos diferenciados (BRASIL, 2012). O atendimento realizado pela UBS Fluvial do município está de acordo com a PNAB, pois trata-se de uma ação direta do governo e as ações e atendimentos realizados são preconizados pelo Ministério da Saúde.

Ter um bom planejamento é fundamental ao prestamos assistência à saúde das populações rurais. Devido as peculiaridades da região amazônica, é preciso organizar previamente tudo que for desafiador para a equipe de saúde que estará isolada, cujo intuito é garantir um atendimento satisfatório e de qualidade. Neste sentido, não basta apenas oferecermos serviços de saúde esporádicos, visto que existe a necessidade de aprimorar o acesso dessas populações à serviços essenciais básicos, especialmente serviços de saúde regulares e contínuos (FRANCO EC, et al., 2015).

Do público alvo que recebeu atendimento nesse período, as mulheres representaram mais de 2/3 desses usuários, um dado que corrobora com diversas pesquisas no campo da saúde, pois as mulheres possuem algumas diferenças fisiológicas em relação aos homens, como por exemplo: a menstruação, contracepção, gravidez, etc. Fatores que podem influenciar diretamente em mais idas aos serviços de saúde. O público feminino também tende a ter mais disciplina em relação a procura por exames periódicos, além de serem gestoras do cuidado da saúde de suas famílias, diante disto, essa informação confirma o que observamos em nosso cotidiano, tendo em vista que existe um grande apelo à diversas campanhas do Ministério da Saúde para que o homem procure e cuide mais da saúde. (GOMES R, et al., 2007; LEVORATO CD, et al., 2014).

De acordo com relatórios apresentados pela equipe de saúde, as hipóteses diagnósticas mais frequentes encontradas foram: verminoses $(25,1 \%)$, intercorrências gástricas $(21,8 \%)$, síndrome gripal $(18,8 \%)$, dor em geral $(16,1 \%)$ (engloba a cefaleia, mialgias, dor de dente, artralgias, etc), intercorrências dermatológicas 
$(9,6 \%)$, infecção do trato urinário $(8,5 \%)$, dentre outras. A ocorrência de verminoses também já foi encontrada em outros estudos envolvendo ribeirinhos (Gama ASM, et al., 2018; Santos FS, et al., 2010), neste caso, o consumo da água não tratada pode ter relação direta com a incidência dessa patologia, além disto, por não possuir estação de tratamento de esgoto nas comunidades, a água utilizada para lavar roupas e tomar banho pode estar contaminada, a ponto de contribuir significativamente para o surgimento de problemas dermatológicos, outra hipótese diagnóstica de grande representação.

Dentre as informações referentes aos atendimentos odontológicos, as exodontias representaram mais da metade de todos os outros procedimentos somados $(54,7 \%)$, um agravo relevante que necessita de intervenções de planejamento e prevenção, a fim de amenizar os elevados indicadores de saúde (AMARAL RC, et al., 2017). Na cidade, o consumo de peixes representa a principal fonte de alimentação dos ribeirinhos, além disto, o pescado também é utilizado como fonte de renda, através da venda direta nas feiras e mercados espalhados pela cidade. Entretanto, na orla e porto do município é normal observar o transporte de alimentos industrializados, cujo destino final é a zona rural.

Alimentos enlatados, processados, tem grande potencial para o surgimento de cáries, pois possuem em suas concentrações elevados níveis de açucares e observa-se que o seu consumo tem aumentado entre todas as faixas etárias da população rural. O baixo nível econômico e educacional também pode estar associado aos altos níveis de cáries e extrações realizados. As dificuldades de acesso aos serviços de saúde, carência de orientações sobre cuidados básicos com a saúde bucal também podem contribuir para 0 surgimento desses agravos (JUNIOR JLAL, et al., 2015; MOURA PG, et al., 2010).

A escuta ativa dos profissionais com os usuários é o elemento central do acolhimento, receber bem os clientes no ambiente de saúde é o primeiro passo para amenizar o desconforto que o usuário apresenta. A Política Nacional de Humanização (PNH) atua a partir de orientações éticas, clínicas, neste sentido, durante essa parte do encontro inicial com o paciente é possível mensurar diversos fatores que fazem parte do contexto de vida de uma determinada população, como por exemplo, a prática da automedicação (BRASIL, 2008; GAMA ASM, 2016).

Durante as viagens, foram observados diversos casos de famílias que procuraram o serviço de saúde apenas com o intuito de receber uma prescrição médica, visando receber determinada medicação para armazenamento domiciliar, vindo a utilizar posteriormente em eventuais necessidades que a família acredita que se encaixe. Uma prática comum na região que pode acarretar diversos riscos à saúde, inclusive já encontrada em estudos envolvendo ribeirinhos, diante disto, cabe aos profissionais intensificarem estratégias para que a educação em saúde seja eficaz, enfatizando os assuntos pertinentes de acordo com os problemas encontrados (GAMA ASM, 2016).

Os comunitários que vivem na área ribeirinha do município possuem carências no acesso à saúde, educação, informação, dentre outras necessidades, além disto, estão expostos às doenças tropicais e ao perigo que os rios vêm oferecendo na última década, em decorrência da ascensão do crime organizado no Solimões. A estratégia de operacionalização da UBS Fluvial trouxe grandes avanços através da implementação dos programas do Ministério da Saúde, neste sentido, fortalecer e avançar nas ações da zona rural é fundamental para a cobertura destes povos. Embora tenha ocorrido melhoria na cobertura de saúde no contexto descrito, é importante ressaltar que para ocorrer maiores avanços é de suma importância o fortalecimento de parcerias intersetoriais.

\section{REFERÊNCIAS}

1. AMARAL RC, et al. A relação entre a saúde bucal e a cárie dentária em oito comunidades ribeirinhas - Pará, Brasil. Rev. Bras. Odontol., Rio de Janeiro, 2017; 74(1): 18-22.

2. BRASIL. Lei $n^{\circ}$ 8.080, de 19 de setembro de 1990. Lei Orgânica da Saúde. Dispõe sobre as condições para a promoção, proteção e recuperação da saúde, a organização e o funcionamento dos serviços correspondentes e dá outras providências. Brasília, set. 1990.

3. BRASIL. Ministério da Saúde. Programa agentes comunitários de saúde (PACS). Brasília: Ministério da Saúde, 2001.

4. BRASIL. Ministério da Saúde. Secretaria de Atenção à Saúde. Núcleo Técnico da Política Nacional de Humanização. Humaniza SUS: documento base para gestores e trabalhadores do SUS. 4. ed. Brasília: Ministério da Saúde, 2008. 
5. MINISTÉRIO DA SAÚDE. Portaria № 2.488, de 21 de outubro de 2011. Aprova a Política Nacional de Atenção Básica, estabelecendo a revisão de diretrizes e normas para a organização da Atenção Básica, para a Estratégia Saúde da Família (ESF) e o Programa de Agentes Comunitários de Saúde (PACS). Diário Oficial [da] República Federativa do Brasil. Brasília: Ministério da Saúde, 2011.

6. MINISTÉRIO DA SAÚDE. Portaria nํ⒉490, de 21 de outubro de 2011. Define os valores de financiamento das Equipes de Saúde da Família Ribeirinhas (ESFR) e custeio das Unidades Básicas de Saúde Fluviais (UBSF), mediante a revisão de diretrizes e normas para a organização da Atenção Básica, instituídos pela Política Nacional de Atenção Básica. Diário Oficial [da] República Federativa do Brasil. Brasília: Ministério da Saúde, 2011.

7. BRASIL. Ministério da Saúde. Política Nacional de Atenção Básica. Brasília: Ministério da Saúde, 2012.

8. FRANCO EC, et al. Promoção da saúde da população ribeirinha da região amazônica: relato de experiência. Rev CEFAC [Internet]. 2015.

9. FRAXE TJP, et al. Comunidades ribeirinhas amazônicas: modos de vida e uso dos recursos naturais. Manaus: EDUA; 2007.

10. GAMA ASM. Automedicação em comunidades ribeirinhas na região do Médio Solimões, Amazonas. [Tese]. São Paulo: Universidade de São Paulo, 2016.

11. GAMA ASM, et al. Inquérito de saúde em comunidades ribeirinhas do Amazonas, Brasil. Cad. Saúde Pública [online]. 2018, 34(2): e00002817.

12. GOMES R, et al. Por que os homens buscam menos os serviços de saúde do que as mulheres? As explicações de homens com baixa escolaridade e homens com ensino superior. Cad. Saude Publica, 2007; 23(3): 565-74.

13. INSTITUTO BRASILEIRO DE GEOGRAFIA E ESTATÍSTICA. Sinopse do censo demográfico de 2010 [Internet].

14. INSTITUTO BRASILEIRO DE GEOGRAFIA E ESTATÍSTICA. Cadastro de municípios localizados na Amazônia Legal [Internet].

15. JUNIOR JLAL, et al. Alimentos x cárie: a ingestão do açúcar em excesso como fator estimulante do desenvolvimento da doença. Ciências biológicas e da saúde. Recife; 2015; 2(2): 11-20.

16. LEVORATO CD, et al. [Factors associated with the demand for health services from a gender-relational perspective]. Cien Saúde Colet [Internet]. 2014.

17. MOURA PG, et al. População indígena: uma reflexão sobre a influência da civilização urbana no estado nutricional e na saúde bucal. Rev. Nutr., Campinas, 2010; 23(3).

18. REIS MHS, et al. Ações de saúde em populações ribeirinhas no interior do estado do Amazonas: Relato de experiência. Em J. C. Schweickardt, Atenção Básica na região amazônica: Saberes e práticas para o fortalecimento do SUS. Porto Alegre: Rede Unida. 2019; 171-181.

19. SANTOS FS, et al. Prevalência de enteroparasitismo em crianças de comunidades ribeirinhas do Município de Coari, no médio Solimões, Amazonas, Brasil. Rev Pan-Amaz Saude. 2010.

20.SOUSA IS. As condições de vida e saneamento nas comunidades da área de influência do gasoduto Coari-Manaus em Manacapuru - AM. Hygeia. 2009; 5(9): 88-98. 